\title{
Expository remarks on topological field theories, branes, complexes and categories
}

\author{
A. A. Bytsenko* \\ Depto. de Física, Univ. Estadual de Londrina, Paraná, Brazil \\ E-mail: abyts@uel.br
}

\section{E. X. Guimarães}

Depto. de Matemática, Univ. de Brasília, DF, Brazil

E-mail: marg@unb.br

\begin{abstract}
Our remarks here are designed to provide members of the Conference, and others, with brief introduction to the sheaves on manifolds, differential complexes, sheaves complexes and categories with its application to topological field theories and D-branes. We shall limit our discussion, in particular, to Chern-Simons and BF theories and some examples of A-brane and B-brane models. This introduction should prepare readers for quite more ambitious discussions found in recent literature. The role of cohomological methods in physical theories will continue to grow as it has grown in past years. In particular it will be an indispensable tool for topological theories of gauge, strings and branes.
\end{abstract}

Fifth International Conference on Mathematical Methods in Physics - IC2006

April 24-28 2006

Centro Brasileiro de Pesquisas Fisicas, Rio de Janeiro, Brazil

\footnotetext{
* Speaker.
} 


\section{Sheaves on manifolds}

A preheaf. A presheaf $\mathscr{F}$ on topological space $X$ consists of the following data:

- For every open set $U \subset X$ one can associate an abelian group $\mathscr{F}(U)$.

- If $V \subset U$ are open sets then a restriction homomorphism occur $r_{U V}: \mathscr{F}(U) \rightarrow \mathscr{F}(V)$.

The following conditions hold: (i) $\mathscr{F}(\emptyset)=0$; (ii) $r_{U U}$ is the identity map; (iii) If $W \subset V \subset U$ then $r_{V W} r_{U V}=r_{U W}$. For the restriction $r_{U V}(\mathscr{P}), \mathscr{P} \in \mathscr{F}(U)$ we use the notation $\left.\mathscr{P}\right|_{V}$. An element of $\mathscr{F}(U)$ is called a section of $\mathscr{F}$ over $U$, while an element of $\mathscr{F}(X)$ is called a global section.

A sheaf. A presheaf $\mathscr{F}$ is called sheaf if for every collection $U_{j}$ of open subsets of $X$ with $U=\bigcup_{j \in I} U_{j}$ the following axioms hold:

A1. If P, Q $\in \mathscr{F}(U)$ and $r_{U U_{j}}(\mathscr{P})=r_{U U_{j}}(\mathscr{Q}) \forall j$, then $\mathscr{P}=\mathscr{Q}$.

$\mathscr{A} 2$. If $\mathscr{P}_{j} \in \mathscr{F}\left(U_{j}\right)$ and if for $U_{j} \cap U_{j} \neq \emptyset, r_{U_{j}, U_{j} \cap U_{\ell}}\left(\mathscr{P}_{j}\right)=r_{U_{\ell}, U_{j} \cap U_{\ell}}\left(\mathscr{P}_{\ell}\right) \forall j$, then there exists an $\mathscr{P} \in \mathscr{F}(U)$ such that $r_{U, U_{j}}(\mathscr{P})=\mathscr{P}_{j}, \forall j .(1.1)$

Let $\mathscr{F}$ and $\mathscr{E}$ be presheaves over $X$, then a morphism of presheaves $\alpha: \mathscr{F} \rightarrow \mathscr{E}$ is a collection of maps $\alpha(U): \mathscr{F}(U) \rightarrow \mathscr{E}(U)$, satisfying the relation $r_{U V} \alpha(U)=\alpha(V) r_{U V}$. Morphisms of sheaves are morphisms of the underlying presheaves.

Coherent sheaves. Let $Y$ be a complex manifold. A sheaf $\mathscr{F}$ over $X$ is called a coherent sheaf of $\mathscr{O}$-modules if for each $x \in X$ there is a neighborhood $U$ of $x$ such that there is an exact sequence of sheaves over $U$ :

$$
\left.\left.\left.\left.0 \longrightarrow \mathscr{F}\right|_{U} \longrightarrow \mathscr{O}^{\oplus p_{1}}\right|_{U} \longrightarrow \mathscr{O}^{\oplus p_{2}}\right|_{U} \longrightarrow \ldots \longrightarrow \mathscr{O}^{\oplus p_{j}}\right|_{U} \longrightarrow 0
$$

\section{Differential complexes}

We recall some definitions of differential complexes to be used in the following. De Rham, Dolbeault and Čech complexes we shall discuss are examples of the differential complex. A direct sum $\mathfrak{C}^{\bullet}=\underset{k \in \mathbb{Z}}{\oplus} \mathfrak{C}^{k}$ of vector spaces $\mathfrak{C}^{k}$ indexed by integers $k$ is called a differential complex if there are homomorphisms

$$
\ldots \longrightarrow \mathfrak{C}^{k-1} \stackrel{\delta}{\longrightarrow} \mathfrak{C}^{k} \stackrel{\delta}{\longrightarrow} \mathfrak{C}^{k+1} \longrightarrow \ldots
$$

such that $\delta^{2}=0$. The homomorphism $\delta$ is called a differential operator of the complex $\mathfrak{C}^{\bullet}$. Elements $c^{k} \in \mathfrak{C}^{k}$ are called $k$-cochains. Let us consider the space

$$
Z^{k}:=\operatorname{Ker} \delta \cap \mathfrak{C}^{k}=\left\{z^{k} \in \mathfrak{C}^{k} \text { such that } \delta z^{k}=0\right\}
$$

Elements $z^{k} \in Z^{k}$ are called $k$-cocycles while $Z^{k}$ is called the space of $k$-cocycles. The space

$$
B^{k}:=\operatorname{Im} \delta \cap \mathfrak{C}^{k}=\left\{b^{k} \in \mathfrak{C}^{k} \text { such that } b^{k}=\delta c^{k-1} \text { for some } c^{k-1} \in \mathfrak{C}^{k-1}\right\}
$$


is called the space of $k$-coboundaries and elements $b^{k} \in B^{k}$ are called $k$-coboundaries. It is evident that $B^{k} \subset Z^{k} \subset \mathfrak{C}^{k}$, indeed each $k$-coboundary $b^{k}$ is a $k$-cocycle: $\delta b^{k}=\delta^{2} c^{k-1}=0$. Cocycles $z^{k}$ such that $z^{k} \neq \delta c^{k-1}$ are called nontrivial $k$-cocycles. The space of nontrivial $k$-cocycles is parametrized by the quotient space $H^{k}:=Z^{k} / B^{k}$. The space $H^{k}$ is called the $k$-th cohomology space of the complex $\mathfrak{C}^{\bullet}$. (For the equivariant cohomology see for example [1].) The cohomology of the differential complex $\mathfrak{C}^{\bullet}$ is the direct sum of the quotient spaces $H^{k}$, and has the form $H^{\bullet}=\underset{k \in \mathbb{Z}}{\oplus} H^{k}$.

De Rham complex. Let $X$ be a differentiable (smooth) manifold of real dimension $n, \Omega^{k}(X)$ is the space of smooth $k$-forms on $X$ and $d$ is an exterior derivative: $\Omega^{k}(X) \rightarrow \Omega^{k+1}(X), d^{2}=0$. Then we have the de Rham complex

$$
\Omega^{\bullet}(X)=\bigoplus_{k=0}^{n} \Omega^{k}(X) .
$$

A differential form $\omega$ on $X$ is called closed if $d \omega=0$. A differential form $\tau$ on $X$ is called exact if $\tau=d \varphi$ for some form $\varphi$. Denote by $Z_{d}^{k}(X)$ the space of closed $k$-forms on $X$ and by $B_{d}^{k}(X)$ the space of exact $k$-forms on $X, B_{d}^{k}(X) \subset Z_{d}^{k}(X) \subset \Omega^{k}(X)$. In the language of differential complexes closed and exact $k$-forms are called de Rham $k$-cocycles and $k$-coboundaries, respectively. The quotient space $H_{d}^{k}(X)=Z_{d}^{k}(X) / B_{d}^{k}(X)$ is called the $k$-th de Rham cohomology space of $X$. The direct sum

$$
H_{d}^{\bullet}(X)=\bigoplus_{k=0}^{n} H_{d}^{k}(X)
$$

is called the de Rham cohomology of $X$.

Dolbeault complex. Let $Y$ be a complex manifold of complex dimension $n$ and $\Omega^{p, q}(Y)$ is the space of smooth $(p, q)$-forms on $Y$. The exterior derivative $d$ on a complex manifold is splitted into a direct sum of two differential operators $\partial$ and $\bar{\partial}$ such that $d=\partial+\bar{\partial}, d^{2}=\partial^{2}=\bar{\partial}^{2}=\partial \bar{\partial}+\bar{\partial} \partial=$ 0 . Differential operators act on the space $\Omega^{p, q}(Y)$ as follows:

$$
\begin{aligned}
& \partial: \Omega^{p, q}(Y) \longrightarrow \Omega^{p+1, q}(Y), \quad \bar{\partial}: \Omega^{p, q}(Y) \longrightarrow \Omega^{p, q+1}(Y), \\
& d: \Omega^{p, q}(Y) \longrightarrow \Omega^{p+1, q}(Y) \oplus \Omega^{p, q+1}(Y) .
\end{aligned}
$$

Consider a sequence of homomorphisms

$$
\Omega^{p, 0}(Y) \stackrel{\bar{\partial}}{\longrightarrow} \Omega^{p, 1}(Y) \stackrel{\bar{\partial}}{\longrightarrow} \ldots \stackrel{\bar{\partial}}{\longrightarrow} \Omega^{p, n}(Y) \longrightarrow 0 .
$$

Since $\bar{\partial}^{2}=0$, the direct sum $\Omega^{p, \bullet}(Y)=\bigoplus_{q=0}^{n} \Omega^{p, q}(Y)$ of the spaces $\Omega^{p, q}(Y)$ is a differential complex, called the Dolbeault complex. A $(p, q)$-form $\omega$ is called $\bar{\partial}$-closed if $\bar{\partial} \omega=0$. In the case $q=0$ such $\bar{\partial}$-closed forms are called holomorphic. Let

$$
Z_{\bar{\partial}}^{p, q}(Y):=\operatorname{Ker} \bar{\partial} \cap \Omega^{p, q}(Y)=\left\{\omega \in \Omega^{p, q}(Y) \text { such that } \bar{\partial} \omega=0\right\}
$$

is the space of $\bar{\partial}$-closed $(p, q)$-forms, i.e. Dolbeault $q$-cocycles in the language of differential complexes, and let

$$
B_{\bar{\partial}}^{p, q}(Y):=\operatorname{Im} \bar{\partial} \cap \Omega^{p, q}(Y)=\left\{\tau \in \Omega^{p, q}(Y), \text { such that } \tau=\bar{\partial} v \text { for some } v \in \Omega^{p, q-1}(Y)\right\}
$$


is the space of $\bar{\partial}$-exact $(p, q)$-forms, i.e. Dolbeault $q$-coboundaries. Denote by $\mathscr{E} p(Y):=Z_{\bar{\partial}}^{p, 0}(Y)$ the space of holomorphic $(p, 0)$-forms. The quotient space $H_{\bar{\partial}}^{p, q}(Y)=Z_{\bar{\partial}}^{p, q}(Y) / B_{\bar{\partial}}^{p, q}(Y)$ is called the $(p, q)$-th Dolbeault cohomology space of $Y$. The direct sum

$$
H_{\bar{\partial}}^{p, \bullet}(Y)=\bigoplus_{q=0}^{n} H_{\bar{\partial}}^{p, q}(Y)
$$

is called the Dolbeault cohomology of $Y$.

Čech complex. We turn to the construction of the Čech cohomology following the lines of the books $[2,3]$. For any smooth $n$-dimensional manifold one can choose an open covering $\mathfrak{U}=\left\{U_{\alpha}\right\}_{\alpha \in I}$ such that each nonempty finite intersection of the open sets $U_{\alpha}$ is diffeomorphic to an open ball in $\mathbb{R}^{n}$ (or biholomorphic to a Stein manifold for the case of complex $n$-manifolds). Such a covering will be called a good covering. For a good covering the Poincaré lemma holds on each finite intersection of the open sets $U_{\alpha}, \alpha \in I$. An ordered collection $\left\langle U_{\alpha_{0}}, \ldots, U_{\alpha_{m}}\right\rangle$ of $m+1$ open sets from the covering $\mathfrak{U}$ such that $U_{\alpha_{0}} \cap \ldots \cap U_{\alpha_{m}} \neq \emptyset$ is called the m-simplex. The set $U_{\alpha_{0} \ldots \alpha_{m}}:=U_{\alpha_{0}} \cap \ldots \cap U_{\alpha_{m}}$ is called a support of the m-simplex $\left\langle U_{\alpha_{0}}, \ldots, U_{\alpha_{m}}\right\rangle$.

Let us consider the space $\mathscr{S}$ of forms of a particular type defined locally on various open sets. Depending on the structure of manifolds (smooth or complex-analytic) this may be the space of smooth $k$-forms, holomorphic $(p, 0)$-forms etc. In other words, we consider various sheaves of forms over manifolds [2,3]. A Čech $m$-cochain $c^{m}$ with values in the space $\mathscr{S}$ is a collection $c^{m}=$ $\left\{c_{\alpha_{0} \ldots \alpha_{m}}\right\}$ of elements $c_{\alpha_{0} \ldots \alpha_{m}}$ from $\mathscr{S}$ defined on supports $U_{\alpha_{0} \ldots \alpha_{m}}$ of $m$-simplex $\left\langle U_{\alpha_{0}}, \ldots, U_{\alpha_{m}}\right\rangle$. The space of Čech $m$-cochains for the covering $\mathfrak{U}$ with values in $\mathscr{S}$ will be denoted by $C^{m}(\mathfrak{U}, \mathscr{S})$. Let us denote by $\rho_{\alpha}$ the restriction operator acting on elements from $\mathscr{S}$ as follows: if $\Sigma \in \mathscr{S}$ is defined on an open set $U$ then $\rho_{\alpha} \Sigma$ is defined on $U \cap U_{\alpha}$. Now letr us consider the map $\delta:=\left\{\rho_{[\alpha \ldots]}\right\}$,

$$
\begin{aligned}
& \delta:\left\{c_{\alpha_{0} \ldots \alpha_{m}}\right\} \longrightarrow\left\{\rho_{\left[\alpha_{0}\right.} c_{\left.\alpha_{1} \ldots \alpha_{m+1}\right]}\right\}, \quad c^{m}=\left\{c_{\alpha_{0} \ldots \alpha_{m}}\right\} \in C^{m}(\mathfrak{U}, \mathscr{S}) \\
& \delta c^{m}=\left\{\rho_{\left[\alpha_{0}\right.} c_{\left.\alpha_{1} \ldots \alpha_{m+1}\right]}\right\} \in C^{m+1}(\mathfrak{U}, \mathscr{S})
\end{aligned}
$$

and $\left[\alpha_{0} \ldots \alpha_{m+1}\right]$ means antisymmetrization with respect to the indices $\alpha_{0}, \ldots, \alpha_{m+1}$. The operator $\delta$ is called a coboundary operator. Since $\rho_{\alpha} \rho_{\beta}=\rho_{\beta} \rho_{\alpha}$, we have $\delta^{2}=0$. Therefore, one can consider a sequence of homomorphisms

$$
C^{0}(\mathfrak{U}, \mathscr{S}) \stackrel{\delta}{\longrightarrow} \ldots \stackrel{\delta}{\longrightarrow} C^{m-1}(\mathfrak{U}, \mathscr{S}) \stackrel{\delta}{\longrightarrow} C^{m}(\mathfrak{U}, \mathscr{S}) \stackrel{\delta}{\longrightarrow} C^{m+1}(\mathfrak{U}, \mathscr{S}) \stackrel{\delta}{\longrightarrow} \ldots
$$

which gives the Čech complex $C^{\bullet}(\mathfrak{U}, \mathscr{S})=\underset{m \geq 0}{\oplus} C^{m}(\mathfrak{U}, \mathscr{S})$. The coboundary operator $\delta$ is a differential operator of this complex in the terminology of differential complexes. Denote by

$$
Z^{m}(\mathfrak{U}, \mathscr{S}):=\operatorname{Ker} \delta \cap C^{m}(\mathfrak{U}, \mathscr{S})=\left\{z \in C^{m}(\mathfrak{U}, \mathscr{S}): \delta z=0\right\}
$$

the space of Čech $m$-cocycles, and by

$$
B^{m}(\mathfrak{U}, \mathscr{S}):=\operatorname{Im} \delta \cap C^{m}(\mathfrak{U}, \mathscr{S})=\left\{b \in C^{m}(\mathfrak{U}, \mathscr{S}): b=\delta c \text { for some } c \in C^{m-1}(\mathfrak{U}, \mathscr{S})\right\}
$$

the space of $\check{C}$ ech $m$-coboundaries. It is evident that $B^{m}(\mathfrak{U}, \mathscr{S}) \subset Z^{m}(\mathfrak{U}, \mathscr{S}) \subset C^{m}(\mathfrak{U}, \mathscr{S})$. Therefore, we can introduce the space $H^{m}(\mathfrak{U}, \mathscr{S})=Z^{m}(\mathfrak{U}, \mathscr{S}) / B^{m}(\mathfrak{U}, \mathscr{S})$ of nontrivial Čech $m$-cocycles, where two distinct elements of $Z^{m}(\mathfrak{U}, \mathscr{S})$ are regarded as equivalent in $H^{m}(\mathfrak{U}, \mathscr{S})$ if they differ by 
a coboundary. We call $H^{m}(\mathfrak{U}, \mathscr{S})$ the $m$-th $\check{C}$ ech cohomology space of the covering $\mathfrak{U}$ with coefficients in the space $\mathscr{S}$. The direct sum

$$
H^{\bullet}(\mathfrak{U}, \mathscr{S})=\underset{m \geq 0}{\oplus} H^{m}(\mathfrak{U}, \mathscr{S})
$$

is called the $\check{C}$ ech cohomology of $\mathfrak{U}$ with coefficients in $\mathscr{S}$. The result depends to some extent on the choice of covering $\mathfrak{U}$, but for a good covering this dependence disappear (for proof see e.g. [2,3]).

Complexes of coherent sheaves. Let $\mathscr{F} \bullet$ denote a complex of coherent sheaves,

$$
\mathscr{F}^{\bullet}: \ldots \stackrel{\delta^{j-2}}{\longrightarrow} \mathscr{F}^{j-1} \stackrel{\delta^{j-1}}{\longrightarrow} \mathscr{F}^{j} \stackrel{\delta^{j}}{\longrightarrow} \mathscr{F}^{j+1} \stackrel{\delta^{j+1}}{\longrightarrow} \ldots
$$

Here $\delta^{j} \delta^{j-1}=0$; for details we refer the reader to the book [12]. Cohomology groups of the complex $\mathscr{F}^{\bullet}$ can be defined as follows: $H^{j}(\mathscr{F} \bullet)=\operatorname{Ker} \delta^{j} / \operatorname{Im} \delta^{j-1}$. Then a morphism of complexes $\alpha: \mathscr{F}^{\bullet} \rightarrow \mathscr{E}^{\bullet}$ induces a morphism of cohomology groups $H(\alpha): H^{\bullet}\left(\mathscr{F}^{\bullet}\right) \rightarrow H^{\bullet}\left(\mathscr{E}^{\bullet}\right)$. A morphism $\alpha$ is said to be a quasi-isomorphism if $H(\alpha)$ is an isomorphism. For homotopy equivalent morphisms $\alpha$ and $\beta$ one gets $H(\alpha)=H(\beta)$.

\subsection{Isomorphisms of cohomologies}

For a differentiable $n$-manifold, we denote by $\Omega^{k}$ the space of locally defined $k$-forms and by $\mathscr{C} \subset \Omega^{0}$ the space of locally constant functions. For a complex $n$-manifold, by $\Omega^{(p, 0)}$ we denote the space of locally holomorphic $(p, 0)$-forms, and by $\mathscr{O}=\Omega^{(0,0)}$ the space of locally holomorphic functions. In other words, we consider the sheaves $\mathscr{C}, \mathscr{O}$ of locally constant functions and locally holomorphic functions respectively, and the sheaves $\Omega^{k}, \Omega^{(p, 0)}$ of forms.

Theorem 1. The de Rham cohomology $H_{d}^{\bullet}(X)$ and $\check{C}$ ech cohomology $H^{\bullet}(X, \mathscr{C})$ of a differentiable $n$-manifold $X$ are isomorphic.

Theorem 2. The Dolbeault cohomology $H_{\bar{\partial}}^{p, \bullet}(Y)$ and $\check{C}$ ech cohomology $H^{\bullet}\left(Y, \Omega^{(p, 0)}\right)$ of a complex n-manifold $Y$ are isomorphic.

For the proof of Theorem 1 and 2 we refer the reader to the book [3].

\section{Abelian theories}

The field-theoretic description of the de Rham and Dolbeault cohomologies can be given respectively by the following action functionals $[4,5]$ :

$$
\begin{aligned}
& S_{\mathrm{dR}}=\int_{X} \sum_{k=1}^{\ell} \omega^{(n-k)} \wedge d \omega^{(k-1)}, \\
& S_{\mathrm{Dol}}=\int_{Y} \sum_{q=1}^{n} \omega^{(n-p, n-q)} \wedge \bar{\partial} \omega^{(p, q-1)},
\end{aligned}
$$


where $\omega^{(s)} \in \Omega^{s}(X)$ are $s$-forms on $X, s=0,1, \ldots, n, \quad \ell=\left[\frac{n+1}{2}\right]$ is the integer part of the number $(n+1) / 2, \omega^{(\ell, s)} \in \Omega^{\ell, s}(Y)$. The Euler-Lagrange equations for these action functionals are

$$
\begin{aligned}
& d \omega^{(k)}=0, \quad k=0,1, \ldots, n-1, \\
& \bar{\partial} \omega^{(p, q-1)}=0, \quad \bar{\partial} \omega^{(n-p, n-q)}=0, \quad q=1, \ldots, n .
\end{aligned}
$$

Solutions of equations associated with de Rham action are elements from $Z_{d}^{k}(X)$. Exact forms from $B_{d}^{k}(X)$ give trivial solutions. Therefore, the space of nontrivial solutions (moduli space) is given by the space $\underset{k=0}{n-1} H_{d}^{k}(X)$. The de Rham theory generalizes Abelian Chern-Simons theory and Abelian topological BF theory defined respectively by the actions

$$
\begin{aligned}
& S_{\mathrm{ACS}}=\int_{X_{3}} A \wedge d A, \\
& S_{\mathrm{ABF}}=\int_{X} B \wedge F,
\end{aligned}
$$

where $A:=\omega^{(1)} \in \Omega^{1}\left(X_{3}\right), \operatorname{dim}_{\mathbb{R}} X_{3}=3, B:=\omega^{(n-2)} \in \Omega^{n-2}(X), F:=d \omega^{(1)}$, and $\omega^{(1)} \in \Omega^{1}(X)$. As an example, one can compare $S_{\mathrm{ACS}}$ with $S_{\mathrm{dR}}$ for $n=3: S_{\mathrm{dR}}=\int_{M}\left(\omega^{(2)} \wedge d \omega^{(0)}+\omega^{(1)} \wedge d \omega^{(1)}\right)$. Let us consider a complex $n$-manifold $Y$. The moduli space of solutions is a vector space which has the form $\bigoplus_{q=1}^{n}\left(H_{\bar{\partial}}^{p, q-1}(Y) \oplus H_{\bar{\partial}}^{n-p, n-q}(Y)\right)$. The Dolbeault theory described by $S_{\text {Dol }}$ generalizes Abelian holomorphic BF theory with the action functional

$$
S_{\mathrm{AhBF}}=\int_{Y} B^{n, n-2} \wedge F^{0,2}
$$

where $B^{n, n-2}:=\omega^{(n, n-2)} \in \Omega^{n, n-2}(Y), F^{0,2}=\bar{\partial} \omega^{(0,1)}, \omega^{(0,1)} \in \Omega^{0,1}(Y)$.

\section{Non-Abelian theories}

There exist non-quadratic topological field theories for which partition functions can be exactly determined as well-defined quantities without actually having to evaluate the functional integrals. It happen when the theory is endowed with a lot of symmetry. Symmetries lead to constraints on the structure of the theory after quantisation. If there is enough symmetry then the structure of the quantum theory may completely determined by the constraints. ${ }^{1}$ A topological gauge theory is by definition endowed with an abundance of symmetry: it is invariant under both gauge transformations and space diffeomorphisms. It has been realised that in the case of the ChernSimons gauge theory the resulting constraints on the structure of the quantised theory are strong enough to completely determine the partition function and expectation values of the Wilson loop observables without actually having to evaluate the functional integrals in general. ${ }^{2}$

Chern-Simons theory. Let $\mathfrak{U}=\left\{U_{\alpha}\right\}$ be a good covering of oriented smooth 3-manifold $X$, $G$ a matrix Lie group, and $\mathfrak{g}$ its Lie algebra. Denote by $A$ a connection 1-form on a (topologically

\footnotetext{
${ }^{1}$ A classic example where this happens is in the two-dimensional WZW conformal field theory. In fact there the constraints resulting from the conformal symmetry completely determine the structure of the quantum theory, in particular the fusion rules.

${ }^{2}$ The possibility that topological invariants could be obtained via Chern-Simons gauge theory had been discussed in [6].
} 
trivial) principal $G$-bundle $P$ over $X$. For such bundles $A$ is a $\mathfrak{g}$-valued 1-form on $X$. Consider the action functional of non-Abelian topological Chern-Simons (CS) theory

$$
S_{\mathrm{CS}}(A)=\frac{1}{4 \pi} \int_{X} \operatorname{Tr}\left(A \wedge d A+\frac{2}{3} A \wedge A \wedge A\right)
$$

field equations have the form $F_{A} \equiv d A+A \wedge A=0$. In the Chern-Simons gauge theory the fields are the $S U(N)$ gauge fields on a general closed oriented 3-manifold $X$, i.e. the 1-forms on $X$ with values in the Lie algebra $s u(N)$. (More general gauge groups can also be considered.) Solutions $A$ define flat connections on $P$, i.e. differential operators $d_{A}=d+A$ such that $d_{A}^{2}=0$.

Analogously, non-Abelian holomorphic Chern-Simons theories are defined on complex 3manifolds and describe flat (0,1)-connections (holomorphic structures). Solutions $A$ can be considered as de Rham 1-cocycles in the non-Abelian de Rham cohomology. On each open set $U_{\alpha}$ equations are solved trivially: $A=\left\{A_{\alpha}\right\}, A_{\alpha}=f_{\alpha}^{-1} d f_{\alpha}$, where $f=\left\{f_{\alpha}\right\}$ is a collection of smooth $G$-valued functions on $\left\{U_{\alpha}\right\}$. To obtain a global solution $A$ on $X$ from local solutions $A_{\alpha}=f_{\alpha}^{-1} d f_{\alpha}$, one should solve the differential equations $f_{\alpha \mid \beta}^{-1} d f_{\alpha \mid \beta}-f_{\beta \mid \alpha}^{-1} d f_{\beta \mid \alpha}=0, f_{\alpha \mid \beta}:=h_{\beta} f_{\alpha}$, on each intersection $U_{\alpha} \cap U_{\beta} \neq \emptyset$, which simply mean that $h_{\beta} A_{\alpha}=h_{\alpha} A_{\beta}$ on $U_{\alpha} \cap U_{\beta} \neq \emptyset$. These equations are equivalent to the equations $d\left(f_{\alpha \mid \beta} f_{\beta \mid \alpha}^{-1}\right)=0$. We see that $c_{\alpha \beta}:=f_{\alpha \mid \beta} f_{\beta \mid \alpha}^{-1}$ is a locally constant $G$-valued function defined on $U_{\alpha} \cap U_{\beta}$. The collection $\left\{c_{\alpha \beta}\right\}$ of $G$-valued functions is a Čech 1-cocycle in the non-Abelian Čech cohomology [7-10], where the cocycle conditions are $\left(h_{\gamma} c_{\alpha \beta}\right)\left(h_{\alpha} c_{\beta \gamma}\right)\left(h_{\beta} c_{\gamma \alpha}\right)=1$ on $U_{\alpha} \cap U_{\beta} \cap U_{\gamma} \neq \emptyset$. Therefore, solutions of field equations $d A+A \wedge A=0$ can be obtained by splitting locally constant $G$-valued functions $c_{\alpha \beta}$, satisfying cocycle conditions, into a product of two smooth $G$-valued functions $f_{\alpha}$ and $f_{\beta}^{-1}$, defined on $U_{\alpha}$ and $U_{\beta}$, respectively. Then, by virtue of the de Rham-Čech correspondence, a collection $\left\{f_{\alpha}^{-1} d f_{\alpha}\right\}=: A$ gives a global solution to field equations.

Topological BF theories. A generalization of Chern-Simons theories to arbitrary dimensions is given by (topological) BF theories. The action functional for non-Abelian topological BF theory has the following form:

$$
S_{\mathrm{BF}}=\int_{X} \operatorname{Tr}\left(B \wedge F_{A}\right),
$$

where $F_{A}$ is the curvature of a connection 1 -form $A$ on a topologically trivial principal $G$-bundle $P$ over $X$, and $B$ is a $\mathfrak{g}$-valued $(n-2)$-form on $X$. The variation of the action with respect to $B$ gives the Chern-Simons field equations, while variation with respect to $A$ gives the equations $d_{A} B=d B+A \wedge B-B \wedge A=0$. Thus, topological BF theories describe flat connections $d_{A}$ on a bundle $P$ over $X$ and $\mathfrak{g}$-valued $d_{A}$-closed $(n-2)$-forms $B$ on $X$. Differential Cher-Simons field equations are equivalent to functional cocycle equations which are solved by $c_{\alpha \beta}=f_{\alpha \mid \beta} f_{\beta \mid \alpha}^{-1}$ for some smooth $G$-valued functions $\left\{f_{\alpha}\right\}$, and for a flat connection $A=\left\{f_{\alpha}^{-1} d f_{\alpha}\right\}$. Equations $d_{A} B=$ $d B+A \wedge B-B \wedge A=0$ can be easily reduced to equations $d\left(f B f^{-1}\right)=0$ from standard de Rham cohomology.

Let $Y$ be a complex $n$-dimensional manifold, $G$ a complex matrix Lie group, $\mathfrak{g}$ its Lie algebra, $P$ a topologically trivial principal $G$-bundle over $Y, A$ a connection 1-form on $P$, and $F_{A}=d A+A \wedge A$ its curvature. Consider holomorphic BF theories with the action functional

$$
S_{\mathrm{hBF}}=\int_{Y} \operatorname{Tr}\left(B^{n, n-2} \wedge F_{A}^{0,2}\right)
$$


where $B^{n, n-2}$ is a $\mathfrak{g}$-valued $(n, n-2)$-form on $Y$ and $F_{A}^{0,2}$ is the $(0,2)$-component of the curvature tensor $F_{A}=F_{A}^{2,0}+F_{A}^{1,1}+F_{A}^{0,2}$. The field equations are $\bar{\partial} A^{0,1}+A^{0,1} \wedge A^{0,1}=0, \bar{\partial} B^{n, n-2}+A^{0,1} \wedge$ $B^{n, n-2}-B^{n, n-2} \wedge A^{0,1}=0$, where $A^{0,1}$ is the $(0,1)$-component of a connection 1-form $A=A^{1,0}+A^{0,1}$ on $P$. If a representation of $G$ in the complex vector space $\mathbb{C}^{\ell}$ is given, we can associate with $P$ the complex vector bundle $E=P \times{ }_{G} \mathbb{C}^{\ell}$ and use vector bundles in the description of BF theories.

It follows from field equations that models (3.6) describe flat $(0,1)$-connections $\bar{\partial}_{A}=\bar{\partial}+A^{0,1}$ on $G$-bundles over complex $n$-manifolds $Y$ and $\bar{\partial}_{A}$-closed $\mathfrak{g}$-valued $(n, n-2)$-forms $B^{n, n-2}$ on $Y$.

One can consider a procedure of constructing solutions to field equations and mapping solutions into one another (dressing transformations) [10]. This cohomological method of solving field equations is based on the equivalence of Čech and Dolbeault descriptions of holomorphic bundles and is a generalization to arbitrary dimensions of the dressing approach (Riemann-Hilbert problems) to solving integrable equations in two dimensions.

Remark 1. The de Rham-Čech isomorphism reduces differential equations $d \omega^{(k)}=0$ on $k$ forms $\omega^{(k)}$ (de Rham $k$-cochains) on a smooth $n$-manifold $X$ to the functional equations $\rho_{\left[\alpha_{0}\right.} c_{\left.\alpha_{1} \ldots \alpha_{k+1}\right]}$ $=0$ on Čech $k$-cochains $\left\{c_{\alpha_{0} \ldots \alpha_{k}}\right\}$ from $C^{k}(\mathfrak{U}, \mathscr{S})$ defined on open subsets $\left\{U_{\alpha_{0} \ldots \alpha_{k}}\right\}$ of $X$. Thus, solutions of topological Chern-Simons and BF theories can be constructed by means of Čech cocycles. Symmetries of these theories can be described in terms of Čech 1-cocycles with values in the sheaf of locally constant maps of the space $X$ into the Lie group $G$.

The Dolbeault-Čech isomorphism reduces differential equations of motion on $(p, q)$-forms (Dolbeault $q$-cochains), defined on a complex $n$-manifold $Y$, to functional equations on Čech $q$ cochains from $C^{q}\left(\mathfrak{U}, \mathscr{E}^{p}\right)$ defined on open subsets $\left\{U_{\alpha_{0} \ldots \alpha_{q}}\right\}$ of $Y$. By using this isomorphism, one obtains another description of solutions of holomorphic Chern-Simons and BF theories.

Non-Abelian cohomological structure. Results of of holomorphic structure of BF theory can be formulate in terms of elements of homological algebra using sheaves of non-Abelian groups. Let $G$ be a (complex) semisimple matrix Lie group, $\mathfrak{g}$ its Lie algebra, $P$ a principal $G-$ bundle over complex $n$-manifold $Y$. Let $\mathscr{F}$ be the sheaf of germs of smooth $G$-valued functions on $Y$ and $\mathscr{E}$ its subsheaf of holomorphic $G$-valued functions. Let $P_{0}$ be a (trivial) $G$-bundle over $Y$ and $\mathscr{A}^{0,1}$ the sheaf of flat $(0,1)$-connections on $P_{0}$ (germs of solutions to the equation of motions of (4.3) for the $(0,1)$-component of a connection 1 -form on $P)$.

Let us introduce the sets of the following quatity: $C^{0}(\mathfrak{U}, \mathscr{F})$ of 0 -cochains of the covering $\mathfrak{U}=\left\{U_{\alpha}\right\}$ of a manifold $Y$ with values in $\mathscr{F}, Z^{0}(\mathfrak{U}, \mathscr{F})$ of 0 -cocycles with values in $\mathscr{F}, C^{1}(\mathfrak{U}, \mathscr{F})$ of 1 -cochains with values in $\mathscr{F}, Z^{1}(\mathfrak{U}, \mathscr{F})$ of 1 -cocycles of the covering $\mathfrak{U}$ with values in the sheaf $\mathscr{F}$ and the 1 -cohomology $H^{1}(\mathfrak{U}, \mathscr{F})$. It is clear that these sets contain the subsets $C^{0}(\mathfrak{U}, \mathscr{E})$, $Z^{0}(\mathfrak{U}, \mathscr{E}), C^{1}(\mathfrak{U}, \mathscr{E}), Z^{1}(\mathfrak{U}, \mathscr{E})$ and $H^{1}(\mathfrak{U}, \mathscr{F})$ respectively (for definitions we refer the reader to the book [11]). By definition $H^{0}(Y, \mathscr{F})=Z^{0}(\mathfrak{U}, \mathscr{F}), H^{0}\left(Y, \mathscr{A}^{0,1}\right)=Z^{0}\left(\mathfrak{U}, \mathscr{A}^{0,1}\right)$. One can choose a covering $\mathfrak{U}=\left\{U_{\alpha}\right\}$ such that $H^{1}(\mathfrak{U}, \mathscr{F})=H^{1}(Y, \mathscr{F}), H^{1}(\mathfrak{U}, \mathscr{E})=H^{1}(Y, \mathscr{E}) .{ }^{3}$ Then some of spaces and groups are defined as follows: $\mathscr{M} \simeq H^{0}\left(Y, \mathscr{A}^{0,1}\right) / H^{0}(Y, \mathscr{F})$, where $\mathscr{M}$ is the moduli space of flat $(0,1)$-connections $A^{0,1}$ parametrizing holomorphic structures of the $(0,1)$-part of

\footnotetext{
${ }^{3}$ This is realized for instance when $U_{\alpha}$ 's are Stein manifolds.
} 
exterior derivative on the bundle $P_{0}$. One can construct the exact sequence of sheaves [7-9]

$$
e \longrightarrow \mathscr{E} \stackrel{\sigma}{\longrightarrow} \mathscr{F} \stackrel{\bar{\delta}^{0}}{\longrightarrow} \mathscr{A}^{0,1} \stackrel{\bar{\delta}^{1}}{\longrightarrow} e,
$$

where $\sigma: \mathscr{E} \rightarrow \mathscr{F}$ is an embedding of $\mathscr{E}$ into $\mathscr{F}, \bar{\delta}^{0}: \mathscr{F} \rightarrow \mathscr{A}^{0,1}$ is a map given for any open set $U$ of the space $Y, \bar{\delta}^{1}$ maps any flat $(0,1)$-connection into zero, and $e$ is a marked element of the considered sets, i.e. identity in the sheaf $\mathscr{E} \subset \mathscr{F}$ and zero in the sheaf $\mathscr{A}^{0,1}$. Then the exact sequence of cohomology sets holds

$$
e \longrightarrow H^{0}(Y, \mathscr{E}) \stackrel{\sigma_{*}}{\longrightarrow} H^{0}(Y, \mathscr{F}) \stackrel{\bar{\delta}_{*}^{0}}{\longrightarrow} H^{0}\left(Y, \mathscr{A}^{0,1}\right) \stackrel{\bar{\delta}_{*}^{1}}{\longrightarrow} H^{1}(Y, \mathscr{E}) \stackrel{\lambda}{\longrightarrow} H^{1}(Y, \mathscr{F}),
$$

where the map $\lambda$ coincides with the canonical embedding by the embedding of sheaves $\sigma: \mathscr{E} \rightarrow \mathscr{F}$. The kernel Ker $\lambda=\lambda^{-1}(e)$ of the map $\lambda$ coincides with a subset of equivalence classes of topologically trivial holomorphic bundles $P$. The following result holds [10]: $\mathscr{M} \simeq H^{0}\left(Y, \mathscr{A}^{0,1}\right) / H^{0}(Y, \mathscr{F})$ $\simeq \operatorname{Ker} \lambda$.

\section{Categories}

A category $\mathscr{C}$ consists of the following data:

- A class $\mathrm{Ob} \mathscr{C}$ of objects $A, B, C, \ldots$;

- A family of disjoint sets of morphisms $\operatorname{Hom}(A, B)$, one for each ordered pair $A, B$ of objects;

- A family of maps $\operatorname{Hom}(A, B) \times \operatorname{Hom}(B, C) \rightarrow \operatorname{Hom}(A, C)$, one for each ordered triplet $A, B$, $C$ of objects.

These data obey the axioms:

$\mathscr{A} 3$. If $f: A \rightarrow B, g: B \rightarrow C, h: C \rightarrow D$, then composition of morphisms is associative, that is, $h(g f)=(h g) f$.

$\mathscr{A} 4$. To each object $B$ there exists a morphism $1_{B}: B \rightarrow B$ such that $1_{B} f=f, g 1_{B}=g$ for $f: A \rightarrow B$ and $g: B \rightarrow C$.

Additive category. An additive category is a category in which each set of morphisms $\operatorname{Hom}(A, B)$ has the structure of an abelian group. The following axioms hold:

$\mathscr{A}$ 5. Composition of morphisms is distributive: $\left(g_{1}+g_{2}\right) f=g_{1} f+g_{2} f, \quad h\left(g_{1}+g_{2}\right)=$ $h g_{1}+h g_{2}$ for any $g_{1}, g_{2}: B \rightarrow C, f: A \rightarrow B, h: C \rightarrow D$.

$\mathscr{A} 6$. There is a null object 0 such that $\operatorname{Hom}(A, 0)$ and $\operatorname{Hom}(0, A)$ consist of one morphism for any $A$.

$\mathscr{A} 7$. To each pair of objects $A_{1}$ and $A_{2}$ there exists an object $B$ and four morphisms $A_{1} \stackrel{j_{1}}{\rightarrow}$ $B \stackrel{\ell_{2}}{\rightarrow} A_{2} \stackrel{j_{2}}{\rightarrow} B \stackrel{\ell_{1}}{\rightarrow} A_{1}$, which satisfy the identities $\ell_{k} j_{k}=1_{A_{k}},(k=1,2), j_{1} \ell_{1}+j_{2} \ell_{2}=1_{b}, \ell_{2} j_{1}=$ $\ell_{1} j_{2}=0$.

Abelian category. It is an additive category $\mathbf{A}$ which satisfies the additional axiom:

$\mathscr{A}$ 8. To each morphism $f: A \rightarrow B$ there exists the sequence $K \stackrel{k}{\rightarrow} A \stackrel{i}{\rightarrow} I \stackrel{j}{\rightarrow} B \stackrel{c}{\rightarrow} K^{\prime}$ with the properties: $j i=f ; K$ is a kernel of $f, K^{\prime}$ is a cokernel of $f ; I$ is a cokernel of $k$ and a kernel of $c$. 
The category of coherent sheaves is the abelian category $\mathbf{A}$.

The derived category. The definition of derived category $\mathbf{D}(\mathbf{A})$ proceeds as follows [12] (see also [13]):

- We begin with category of complexes of coherent sheaves $\operatorname{Kom}(\mathbf{A}): \operatorname{Ob} \operatorname{Kom}(\mathbf{A})=$ $\left\{\right.$ complexes $B^{\bullet}$ of coherent sheaves $\} ; \operatorname{Hom}\left(B^{\bullet}, C^{\bullet}\right)=$ morphisms of complexes $B^{\bullet} \rightarrow C^{\bullet}$.

- The homotopy category $\mathbf{K}(\mathbf{A})$ can be determined as follows: Ob $\mathbf{K}(\mathbf{A})=\mathrm{Ob} \operatorname{Kom}(\mathbf{A})$, Mor $\mathbf{K}(\mathbf{A})=\operatorname{Mor} \operatorname{Kom}(\mathbf{A})$ modulo homotopy equivalence.

- Finally the derived category $\mathbf{D}(\mathbf{A})$ is determined as follows: $\mathrm{Ob} \mathbf{D}(\mathbf{A})=\mathrm{Ob} \mathbf{K}(\mathbf{A})$.

The morphisms of $\mathbf{D}(\mathbf{A})$ are obtained from morphisms in $\mathbf{K}(\mathbf{A})$ by inverting all quasi-isomorphisms. The derived category $\mathbf{D}(\mathbf{A})$ is the additive category.

\section{Topological D-branes}

Let us address to a specific set of topological field theories with target space $X$. The sum of all such topological field theories gives $\mathbf{D}(X)$. The objects in $\mathbf{D}(X)$ is called topological branes [16]. These topological field theories can be build by making use the following processes:

- Take into account Witten's B-model $[14,15]$.

- Add the notion of integral grading [16].

- Construct specific kinds of marginal deformations of this theory to form a more general class of objects.

The dynamics of off-shell open string modes in the topological B-model is captured the holomorphic Chern-Simons action which defines a cubic string field theory. In order to write down this action, we must regard a holomorphic bundle $E$ on $Y$ as a $C^{\infty}$ vector bundle equipped with a connection $A$ so that $F^{0,2}(A)=0$. The off-shell boundary fields form an associative algebra $\mathfrak{A}=\oplus_{p=0}^{3} \Omega^{0, p}(Y, \operatorname{End}(E))$, where $p$ represents the ghost number. A morphism $\bar{\partial}_{A}$ represents the BRST operator $Q$ acting on off-shell states, which defines a structure of differential graded algebra on $\mathfrak{A}$. The massless spectrum of the theory is parameterized by the graded vector space

$H=\oplus_{p=0}^{3} H^{0, p}(Y, \operatorname{End}(E))$, and the boundary chiral ring structure is defined by the Yoneda pairing on $H$. In addition the physical on-shell operators in string field theory correspond to elements of degree one in $H$, that is cohomology classes in $H^{0,1}(Y$, End $(E))$.

It has been proved that the sum of a certain class of topological field theories on an algebraic variety $X$ is equivalent (in some sense) to the bounded derived category of coherent sheaves (see, for example, the review paper [18]). The finite collection of nontrivial sheaves $\mathscr{E} n$ is the objects in our category. We can say that the objects are D-branes and the morphisms are open strings. Composition of morphisms is then given by the Yoneda pairing [19]:

$$
\operatorname{Ext}^{p}\left(\mathscr{E}^{\ell}, \mathscr{E}^{m}\right) \otimes \operatorname{Ext}^{q}\left(\mathscr{E}^{m}, \mathscr{E}^{n}\right) \longrightarrow \operatorname{Ext}^{p+q}\left(\mathscr{E}^{\ell}, \mathscr{E}^{n}\right)
$$


which means that two open strings joining along a common boundary $\mathscr{E}^{m}$. There are the following conditions that these objects and morphisms need to satisfy in order to be a category: For every object there exists an identity morphism which is given by $1_{n} \in \operatorname{Ext}^{0}\left(\mathscr{E}^{n}, \mathscr{E}^{n}\right)$; the morphisms are associative.

The content of a topological field theory is given completely by its operator algebra. The topological field theory we are discussing is therefore completely equivalent to this category. By our construction we have described a category with a finite number of objects. The category $\mathbf{T}(X)$ of all possible topological D-branes on $X$ has an infinite number of objects. Thus, the category associated to a particular topological field theory is a subcategory of $\mathbf{T}(X)$.

The open strings vertex operators (which deform the theory) must be ghost number one. Let us consider a theory with a single D-brane which is given by the locally free sheaf $\mathscr{E}$. Then the ghost number one we have $\operatorname{Ext}^{1}(\mathscr{E}, \mathscr{E})$. Ignoring potential obstructions in the moduli space this agrees with the expected deformations of the sheaf $\mathscr{E}(\mathscr{E}$ can be associated with a vector bundle $E$, in that case $\left.\operatorname{Ext}^{1}(\mathscr{E}, \mathscr{E})=H^{1}(X, \operatorname{End}(E))\right)$. Let a open string stretching between two possibly distinct D-branes. It can be done assuming that each $\mathscr{E}^{n}$ is actually a direct sum of two sheaves for all $n$, i.e. the sum $\mathscr{E}^{n} \oplus \mathscr{F}^{n}$. We can restrict the maps in this complex for a particular string to being block-diagonal: $\delta_{n}^{E}: \mathscr{E}^{n} \rightarrow \mathscr{E}^{n+1}, \delta_{n}^{F}: \mathscr{F}^{n} \rightarrow \mathscr{F}^{n+1}, \delta^{2}=0$ and with no $\delta$ maps mixing the $\mathscr{E}$ 's and $\mathscr{F}$ 's. Then we have two D-branes; one associated to $\mathscr{E}^{\bullet}$ and one associated to $\mathscr{F} \bullet$. The topological field theory is specified by a complex of locally free sheaves:

$$
\mathscr{E} \bullet\left(\mathscr{F}^{\bullet}\right): \ldots \longrightarrow \mathscr{E}^{-1}\left(\mathscr{F}^{-1}\right) \stackrel{\delta_{-1}^{E}\left(\delta_{-1}^{F}\right)}{\longrightarrow} \mathscr{E}^{0}\left(\mathscr{F}^{0}\right) \stackrel{\delta_{0}^{E}\left(\delta_{0}^{F}\right)}{\longrightarrow} \mathscr{E}^{1}\left(\mathscr{F}^{1}\right) \stackrel{\delta_{1}^{E}\left(\delta_{1}^{F}\right)}{\longrightarrow} \ldots
$$

The original vector bundle $E$ is finite-dimensional, thus this complex is bounded. Let us consider the sheaf given by $\mathscr{H} o m\left(\mathscr{E}^{m}, \mathscr{F}^{n}\right)$. The vector spaces $\operatorname{Hom}\left(\mathscr{E}^{m}, \mathscr{F}^{n}\right)$ is difer from the sheafs $\mathscr{H} o m\left(\mathscr{E}^{m}, \mathscr{F}^{n}\right)$ which associates an open set $U$ with local holomorphic maps from sections of the bundle $E^{m}$ over $U$ to sections of the the bundle $F^{n}$ over $U$. The maps $\delta_{n}^{E}$ and $\delta_{n}^{F}$ induce a double complex:

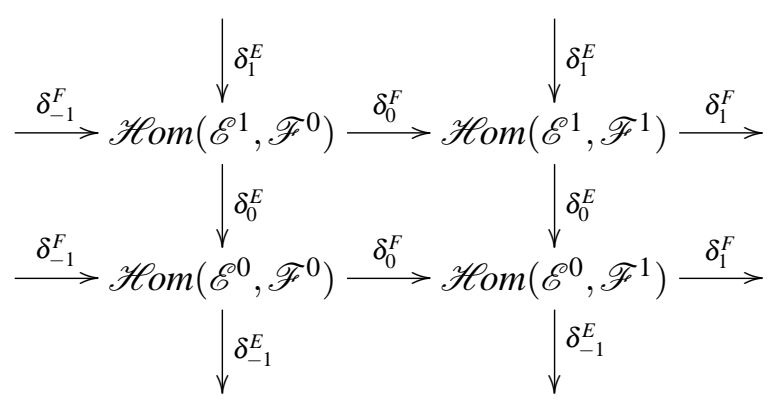

while the single complex has the form

$$
\cdots \longrightarrow \mathscr{H}^{0}{ }^{0}(\mathscr{E} \bullet, \mathscr{F} \bullet) \stackrel{\bar{\delta}_{0}}{\longrightarrow} \mathscr{H}{ }^{1}(\mathscr{E} \bullet, \mathscr{F} \bullet) \stackrel{\bar{\delta}_{1}}{\longrightarrow} \cdots
$$

Here $\mathscr{H}_{o m^{q}}\left(\mathscr{E} \bullet, \mathscr{F}^{\bullet}\right)=\oplus_{n} \mathscr{H} o m\left(\mathscr{E}^{n}, \mathscr{F}^{n+q}\right), \bar{\delta}=\delta^{E}+\delta^{F}$, and $\delta^{E}$ and $\delta^{F}$ anti-commute. The operator product is a generalization of the Yoneda pairing $\operatorname{Hom}^{P}\left(\mathscr{E}^{\bullet}, \mathscr{F}^{\bullet}\right) \otimes \operatorname{Hom}^{Q}\left(\mathscr{F}^{\bullet}, \mathscr{G}^{\bullet}\right) \rightarrow$ $\operatorname{Hom}^{P+Q}\left(\mathscr{E} \bullet, \mathscr{G}^{\bullet}\right)$. Taking into account a decomposition $\mathscr{E}=\oplus_{n \in \mathbb{Z}} \mathscr{E}^{n}$, where $\mathscr{E}^{n}$ is a B-brane with ghost number $n$, we may construct a general collection of D-branes in terms of a locally-free 
sheaf $\mathscr{E}$. In addition the ghost number one operators in this B-model are therefore elements of $\operatorname{Ext}^{k}\left(\mathscr{E}^{n}, \mathscr{E}^{n-k+1}\right)$ for any $n$ and $k$.

\section{Deformations in category}

Remark 2. A functor $\mathrm{F}$ from category $\mathbf{A}$ to category $\mathbf{B}$ associates from each object $A$ of $\mathbf{A}$ object $\mathrm{F}(A)$ from $\mathbf{B}$ and from each morphism $\alpha: A \rightarrow B$ of $\mathbf{A}$ morphism $\mathrm{F}(\alpha): \mathrm{F}(A) \rightarrow \mathrm{F}(B)$ of $\mathbf{B}$ such that $\mathrm{F}$ has the composition and identity morphisms: $\mathrm{F}(\alpha \circ \beta)=\mathrm{F}(\alpha) \circ \mathrm{F}(\beta), \mathrm{F}\left(1_{A}\right)=1_{\mathrm{F}(A)}$. If functor has properties $\mathrm{F}(A) \rightarrow \mathrm{F}(B)$ and $\mathrm{F}(B) \rightarrow \mathrm{F}(A)$ then it called the covariant functor.

Remark 3. A chain map which induces an isomorphism on the cohomology groups of the complex is called a quasi-isomorphism.

Theorem 3. Let $\mathbf{A}$ be an abelian category, and let $\operatorname{Kom}(\mathbf{A})$ be the category of complexes over $\mathbf{A}$. There exists a category $\mathbf{D}(\mathbf{A})$ and a functor $\mathrm{Q}: \mathbf{K o m}(\mathbf{A}) \rightarrow \mathbf{D}(\mathbf{A})$ such that the following properties hold:

- For any quasi-isomorphism $f, \mathrm{Q}(f)$ is an isomorphism.

- Any functor $\mathbf{F}: \operatorname{Kom}(\mathbf{A}) \rightarrow \mathbf{B}$ transforming quasi-isomorphisms into isomorphisms can be uniquely factorized through $\mathbf{D}(\mathbf{A})$. It means that there exists a unique functor $\mathrm{G}: \mathbf{D}(\mathbf{A}) \rightarrow \mathbf{B}$ which satisfy $\mathrm{F}=\mathrm{G} \circ \mathrm{Q}$.

Following the lines of [18] let us consider the category $\mathbf{K}_{\mathrm{LF}}(X)$ of complexes of locally free sheaves on $X$ and the morphisms in this category which are chain maps: a chain in $\mathbf{K}_{\mathrm{LF}}(X)$ maps to the corresponding D-brane in $\mathbf{T}(X)$, and a chain map in $\mathbf{K}_{\mathrm{LF}}(X)$ maps to an element of $\operatorname{Hom}^{0}(\mathscr{E} \bullet, \mathscr{F} \bullet)$ in $\mathbf{T}(X)$.

Mapping cone construction. Let $f$ be a quasi-isomorphism between two complexes $f: \mathscr{E} \bullet \rightarrow$ $\mathscr{E} \bullet$. Note that $f^{*}$ is an isomorphism. Indeed, let $\mathscr{E}^{\bullet}\left(\mathscr{F}^{\bullet}\right)$ be acyclic (be a complex with trivial cohomology) and let $\mathscr{F}^{\bullet}\left(\mathscr{E}^{\bullet}\right)$ be any complex. In this case the cohomology of $\operatorname{Hom}^{P}\left(\mathscr{E}^{\bullet}, \mathscr{F}^{\bullet}\right)$ $\left(\operatorname{Hom}^{P}\left(\mathscr{E}^{\bullet}, \mathscr{F}^{\bullet}\right)\right)$ is trivial for any $\mathscr{E}^{\bullet}\left(\mathscr{F}^{\bullet}\right)$. Let us introduce the Cone of a map $f$ of complexes.

Definition 1. The mapping cone $\operatorname{Cone}\left(f: \mathscr{E}_{\bullet} \rightarrow \mathscr{F} \bullet\right)$ can be defined as the complex

$$
\longrightarrow \mathscr{E}^{1} \oplus \mathscr{F}^{0} \stackrel{\left(\begin{array}{cc}
\delta_{E} & 0 \\
f & \delta_{F}
\end{array}\right)}{\longrightarrow} \mathscr{E}^{2} \oplus \mathscr{F}^{1} \stackrel{\left(\begin{array}{cc}
\delta_{E} & 0 \\
f & \delta_{F}
\end{array}\right)}{\longrightarrow} \mathscr{E}^{3} \oplus \mathscr{F}^{2} \longrightarrow \ldots
$$

Cone $\left(f: \mathscr{E}^{\bullet} \rightarrow \mathscr{F} \bullet\right)$ is acyclic iff the map $f$ is a quasi-isomophism. As an example, for chain complex $\mathscr{E} \bullet \oplus \mathscr{E} \bullet[1] \oplus \mathscr{E} \bullet$ we have $\delta=\left(\begin{array}{ccc}\delta_{A} & 0 & 0 \\ - \text { Id } & \delta_{A}[1] & \text { Id } \\ 0 & 0 & \delta_{A}\end{array}\right)$. If $f$ is a quasi-isomorphism then its cone is acyclic. and the groups $\operatorname{Hom}^{P}(\operatorname{Cone}(f), \mathscr{F} \bullet)$ associated to the cone are zero. Thus $f^{*}: \operatorname{Hom}^{P}\left(\mathscr{E} \bullet, \mathscr{F}^{\bullet}\right) \rightarrow \operatorname{Hom}^{P}\left(\mathscr{E}^{\bullet}, \mathscr{F}^{\bullet}\right)$. provides the canonical isomorphism. Therefore a quasiisomorphism in $\mathbf{K}_{\mathrm{LF}}(X)$ maps to an isomorphism in $\mathbf{T}(X)$. Let us take into accout complexes over 
an abelian category, it gives a possibility to use Theorem 3. Consider the category of coherent sheaves (the smoller category of locally-free sheaves is not abelian, since it does not contain its own cokernels). Let as befor $\operatorname{Kom}(X)$ denote the category of bounded complexes of coherent sheaves on $X$. We can defined a functor from $\operatorname{Kom}(X)$ to $\mathbf{T}(X)$, and therefore by Theorem 3 can constructed a functor $\mathrm{G}: \mathbf{D}(X) \rightarrow \mathbf{T}(X)$ (see for details [18]).

\section{Monodromy}

The monodromy acting on the derived category $\mathbf{D}(A)$ and is induced by a so-called FurierMukai transform [16] associated with some generator $K^{\bullet} \in \mathbf{D}(A)$. Action of the monodromy on a complex $\mathscr{B} \bullet$ is given by

$$
\mathscr{B} \bullet \longmapsto \mathbf{R} p_{1 *}\left(K^{\bullet} \stackrel{\mathbf{L}}{\otimes} p_{2}^{*}\left(\mathscr{B}^{\bullet}\right)\right) \text {. }
$$

The projection maps from $X \times X$ to its first and second factors is:

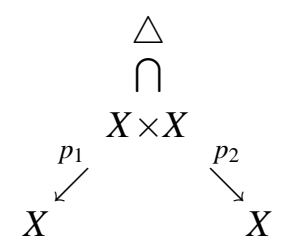

Here $\triangle \subset X \times X$ is the diagonal embedding of $X$. For the monodromy action formula we have used the following transformations:

- Complex of sheaves $\mathscr{B}^{\bullet}$ on $X$ has been pulling back to the inverse-image complex of sheaves $p_{2}^{*}\left(\mathscr{B}^{\bullet}\right)$ on $X \times X$.

- The left-derived complex (hence the capital letter $\mathbf{L}$ ) of sheaves on $X$ has been constructed by taking the tensor-product with the generator $K^{\bullet}$.

- The right-derived complex (hence the capital letter $\mathbf{R}$ ) of sheaves on $X$ has been constructed by pushing-forward to the direct image complex $p_{1}(\bullet)$.

In the case of A-branes monodromy associated with a movement around loops in the moduli space is purely classical. Way around non-contractible loops in the moduli space of complex structure leads to a non-trivial monodromy associated with the periods of the holomorphic 3-form over integral 3-cycles. In that case the monodromy can be interpreted as an automorphism of group $H_{3}(Y, \mathbb{Z})$ which preserves the intersection form between 3-cycles. The homology classes of Abranes undergo monodromy and as a consequence the A-branes themselves undergo monodromy.

In the B-brane case the brane charge is an element of $H^{\text {even }}(X, \mathbb{Z})$. Considering the case of the quintic ${ }^{4}$ one can map between $H_{3}(Y, \mathbb{Z})$ and $H^{\text {even }}(X, \mathbb{Z})$. Therefore the monodromy action from the A-model into the B-model can be copied.

${ }^{4}$ Let, as an example, $Y$ be the quintic hypersurface in $C \mathbb{P}^{4}$. We can defined the mirror $M$ as the orbifold $Y / \mathbb{Z}_{5}^{3}$. Since the virtue of mirror symmetry [20] the Kähler moduli space of $Y$ is identified with the complex structure moduli space of $M$, which is described by the Picard-Fuchs equation: $\left\{\theta_{z}^{4}+5 z \prod_{\ell=4}^{1}\left(5 \theta_{z}+\ell\right)\right\} \omega_{k}(z)=0$. Here the complex variable $z$ spans the complex structurte moduli space $M, \theta_{z}=z d / d z$. Point of the Landau-Ginzburg potential of the moduli space $Y$ is mirror to $z=\infty$. The limit of the large radius of $Y$ is mirror to $s=0$, while the conifold point of $Y$ is mirror to $z=1$. The periods $\omega_{k}(z)$ correspond to these three points are singular. 
As an example, let us begin with a particular sheaf $\mathscr{F}$ and then compute the monodromy action on Chern character $\operatorname{ch}(\mathscr{F})$. We can find examples where the resulting element of $H^{\text {even }}(X, \mathbb{Z})$ cannot correspond to the Chern character of any sheaf. (In the case of hyperbolic geometry some explicit results for the index of a Dirac operator, the Chern character and lowest K-groups, associated with topological charges of branes, the reader can find in [17].) But going to the derived category solves these problems [18]. In category language we have a functor from $\mathbf{D}(X)$ to $\mathbf{D}(X)$ that is a bijection on the (isomorphism classes of) objects and that preserves the corresponding morphisms. Such situation on $\mathbf{D}(X)$ is always induced by a Fourier-Mukai transform.

Monodromy of the quintic. As an example, let us consider a monodromy about the LandauGinsburg point in the Kähler moduli space of the quintic. Such a monodromy is generated by [18]

$$
K_{L G}^{\bullet}=0 \longrightarrow \mathscr{O} \otimes \mathscr{O}(1) \longrightarrow \mathscr{O}_{\triangle}(1) \longrightarrow 0
$$

where the notation $A \otimes B$ to mean $p_{1}^{*} A \otimes p_{2}^{*} B$ for $A, B \in \mathbf{D}(X)$ has been introduced. The explicit monodromy calculations for $\mathscr{O} \in \mathbf{D}(A)$ the reader also can find in [18].

\section{Hochschild cohomology of D-branes category}

The closed string correlators perhaps can be constructed from the open ones using topological string theories as a model. This conjecture has been partially verifyed by means of computation of the Hochschild cohomology of the category of D-branes.

Definition 2. Let $A$ be an associative algebra over $\mathbb{C}$. The Hochschild cochain complex with coefficients in $A$ is the sequence of vector spaces $C^{n}(A)=\operatorname{Hom}_{\mathbb{C}}\left(A^{\otimes n}, A\right), \quad n=0,1, \ldots$, equipped with an operator $\delta: C^{n}(A) \rightarrow C^{n+1}(A)$ defined by

$$
\begin{aligned}
(\delta f)\left(a_{1}, \ldots, a_{n+1}\right) & =a_{1} f\left(a_{2}, \ldots, a_{n}\right)+\sum_{i=1}^{n}(-1)^{i} f\left(a_{1}, \ldots, a_{i-1}, a_{i} a_{i+1}, a_{i+2}, \ldots, a_{n}\right) \\
& +(-1)^{n+1} f\left(a_{1}, \ldots, a_{n}\right) a_{n+1}
\end{aligned}
$$

The cohomology of $\delta$ in degree $n$ will be denoted $\operatorname{HH}^{n}(A)$,

$$
\mathrm{HH}^{n}(A):=\frac{\operatorname{Ker}\left(\delta: C^{n}(A) \longrightarrow C^{n+1}(A)\right)}{\operatorname{Im}\left(\delta: C^{n-1}(A) \longrightarrow C^{n}(A)\right)}
$$

and called the Hochschild cohomology of A with coefficients in A. The connection between Hochschild cohomology and infinitesimal deformations of $A$ is given by $\mathrm{HH}^{2}(A)$. Denote the product in $A$ by $\mathfrak{m}$, an infinitesimally deformed product by $\mathfrak{m}_{t}=\mathfrak{m}+t \varphi$, where $t^{2}=0$, then the map $A^{2} \rightarrow A$ is a Hochschild cocycle and the trivial deformations are given by Hochschild coboundaries. Indeed,

$$
\begin{aligned}
& \left.\mathfrak{m}_{t}\left(\mathfrak{m}_{t}\left(a_{1}, a_{2}\right), a_{3}\right)\right)=a_{1} a_{2} a_{3}+t\left(\varphi\left(a_{1}, a_{2}\right) a_{3}+\varphi\left(a_{1} a_{2}, a_{3}\right)\right), \\
& \mathfrak{m}_{t}\left(a_{1}, \mathfrak{m}_{t}\left(a_{2}, a_{3}\right)\right)=a_{1} a_{2} a_{3}+t\left(a_{1} \varphi\left(a_{2}, a_{3}\right)+\varphi\left(a_{1}, a_{2} a_{3}\right)\right), \\
& \left.a_{1} \varphi\left(a_{2}, a_{3}\right)-\varphi\left(a_{1} a_{2}, a_{3}\right)+\varphi\left(a_{1}, a_{2} a_{3}\right)-\varphi\left(a_{1}, a_{2}\right) a_{3}=0 \text { (since associativity of } \mathfrak{m}_{t}\right) .
\end{aligned}
$$

The last condition is simply the condition $\delta \varphi=0$. A trivial deformation is given by the condition that $A$ with the new multiplication is isomorphic to the original algebra structure. Thus there is 
a linear bijection $\sigma_{t}: A \rightarrow A$ such that $\mathfrak{m}_{t}\left(\sigma_{t}\left(a_{1}\right), \sigma_{t}\left(a_{2}\right)\right)=\sigma_{t}\left(a_{1} a_{2}\right)$. Let $\sigma_{t}:=I+t \lambda$, where $\lambda: A \rightarrow A$ is a linear map. We get

$$
\begin{aligned}
\mathfrak{m}_{t}\left(a_{1}, a_{2}\right) & =\sigma_{t}\left(\sigma_{t}^{-1}\left(a_{1}\right), \sigma_{t}^{-1}\left(a_{2}\right)\right)=a_{1} a_{2}-t\left(a_{1} \lambda\left(a_{2}\right)-\lambda\left(a_{1} a_{2}\right)+\lambda\left(a_{1}\right) a_{2}\right) \\
& =a_{1} a_{2}-t(b \lambda)\left(a_{1}, a_{2}\right) .
\end{aligned}
$$

Therefore coboundaries give rise to trivial deformations. $\mathrm{HH}^{2}(A)$ classifies nontrivial deformations of the associative algebra structure on $A$. The total Hochschild cohomology $\mathrm{HH}^{\bullet}(A)$ classifies infinitesimal deformations of $A$ in the class of $A_{\infty}$ algebras.

Definition 3. Let $A$ be a $\mathbb{Z}_{2}$-graded algebra and $A_{p}$ be a degree-p component of $A$, such that $A_{p}$. $A_{q} \subset A_{p+q}$. We say that element $f$ of $C^{n}(A)$ has an internal degree $p$ if $f\left(a_{1}, \ldots, a_{n}\right) \in A_{p+k_{1}+\cdots+k_{n}}$, $a_{i} \in A_{k_{i}}$. The vector space $C^{n}(A)$ is graded by the internal degree, and the total degree of an element has the form $C^{\bullet}(A)=\oplus_{n} C^{n}(A)$. The Hochschild complex is graded by the total degree, and the Hochschild differential can be expressed in the form

$$
\begin{aligned}
(\delta f)\left(a_{1}, \ldots, a_{n+1}\right) & =(-1)^{a \cdot f} a_{1} f\left(a_{2}, \ldots, a_{n}\right)+\sum_{j=1}^{n}(-1)^{j} f\left(a_{1}, \ldots, a_{j-1}, a_{j} a_{j+1}, a_{j+2}, \ldots, a_{n}\right) \\
& +(-1)^{n+1} f\left(a_{1}, \ldots, a_{n}\right) a_{n+1} .
\end{aligned}
$$

Definition 4. Let $\mathscr{A}=(A, Q)$ be a differential graded algebra (DG-algebra). The degree-1 derivation $Q$ as the map $Q: A_{p} \rightarrow A_{p+1}$ satisfies $Q^{2}=0$, and given by

$$
(Q f)\left(a_{1}, \ldots, a_{n}\right)=Q\left(f\left(a_{1}, \ldots, a_{n}\right)\right)-\sum_{j=1}^{n}(-1)^{v_{1}+\ldots+v_{j-1}+f+n-1} f\left(a_{1}, \ldots, a_{j-1}, Q a_{i}, a_{j+1}, \ldots, a_{n}\right) .
$$

On the bigraded vector space $C^{\bullet}(A)$ there are two comute differentials: $Q$ and $\delta$. Thus the Hochschild cohomology of $\mathscr{A}$ is defined to be the cohomology of $(-1)^{n} Q+\delta$. In the category of DG-algebras $\mathrm{HH}^{\bullet}(\mathscr{A})$ classifies deformations of $(A, Q)$ regarded as an $A_{\infty}$ algebra.

Remark 4. Closed topological string states are related to infinitesimal deformations of the open-string theory. If in the theory exists a single D-brane then all the information is encoded in an associative algebra $A$ equipped with a BRST differential $Q$ of ghost number one (i.e. in DGalgebra) and an invariant scalar product. Equivalence classes of deformations of these data are described by a Hochschild cohomology theory of $(A, Q)$. The conjecture is [21]: The space of physical closed-string states is isomorphic to the Hochschild cohomology of $(A, Q)$.

Let a D-brane be an object of an additive category, and let the space of open strings between two D-branes be the space of morphisms. The algebra of open string states for a particular Dbrane is its endomorphism algebra. Then BRST operators give rise to differentials on all spaces of morphisms, and it is convenient to dealing with a differential graded category. ${ }^{5}$ In order to analyse the space of physical open-string states between two D-branes, one has to know the cohomology of the BRST operator on the space of morphisms. The appropriate cohomology is the Hochschild

\footnotetext{
${ }^{5}$ The grading is given by the ghost number.
} 
cohomology which classifies equivalence classes of deformations of such categories. A heuristic conjecture is: The space of physical closed strings is isomorphic to the Hochschild cohomology of the category of D-branes.

For the topological Landau-Ginzburg models the category of D-branes can be thought of as the category of curved differential graded modules (CDG-modules) over a certain commutative CDG-algebra [22]. The Hochschild cohomology of the category of Landau-Ginzburg branes has been computed in [21]. Note also Landay-Ginzburg models on orbifolds, which can provide an alternative description of certain Calabi-Yau sigma-models (or so-called Gepner models [23]).

\section{References}

[1] A. A. Bytsenko, M. Libine and F. L. Williams, Localization of Equivariant Cohomology for Compact and Non-Compact Group Action, JDSGT 3 (2005) 171 [math.SG/0502190].

[2] R. Bott and L. W. Tu, Differential forms in algebraic topology, Springer, Berlin, 1982.

[3] P. Griffiths and J. Harris, Principles of algebraic geometry, Wiley, New-York, 1978.

[4] A. S. Schwarz, The Partition Function of Degenerate Quadratic Functional and Ray-Singer Invariants, Lett. Math. Phys. 2 (1978) 247.

[5] A. S. Schwarz, The Partition Function of a Degenerate Functional, Commun. Math. Phys. 67 (1979) 1.

[6] A. S. Schwarz, Abstracts (Part II), Baku Int. Topological Conf. Baku 1987.

[7] P. Dedecker, Sur la Cohomologie non Abelienne I (Dimension Deux), Canad. J. Math. 12 (1960) 231.

[8] J. Frenkel, Cohomologie non Abelienne et Espaces Fibres, Bull. Soc. Math. France 85 (1957) 135.

[9] A. L. Oniščik, Certain Contcepts and Applications of the Theory of Nonabelian Cohomologies, Trudi Mosk. Mat. Obsch. 17 (1967) 45 [in Russian].

[10] T. A. Ivanova and A. D. Popov, Dressing Symmetries of Holomorphic BF Theories, J. Math. Phys. 41 (2000) 2604.

[11] F. Hirzebruch, Topological methods in algebraic geometry, Springer, New York, 1966.

[12] S. I. Gelfand and Yu. I. Manin, Homological algebra, Springer-Verlag, Berlin, 1994.

[13] Y. Malyuta, D-Branes and Derived Categories, hep-th/0210057.

[14] E. Witten, Mirror Manifolds and Topological Field Theory, in S.-T. Yau, editor, "Essays on Miror Manifolds", International Press, 1992 [hep-th/9112056].

[15] E. Witten, Chern-Simons Gauge Theory as a String Theory, in H. Hofer et al., editors, "The Floer Memorial Volume", pp. 637-678, Birkhäuser, 1995 [hep-th/9207094].

[16] M. R. Douglas, D-branes, Categories, and N=1 Supersymmetry, J. Math. Phys. 42 (2001) 2818 [hep-th/0011017].

[17] L. Bonora and A. A. Bytsenko, Fluxes, Brane Charges and Chern Morphisms of Hyperbolic Geometry, Class. Quant. Grav. 23 (2006) 3895 [hep-th/0602162].

[18] P. S. Aspinwall, D-Branes on Calabi-Yau Manifolds, hep-th/0403166.

[19] R. Hartshorne, Algebraic Geometry, Graduate Texts in Mathematics 52, Springer-Verlag, 1977. 
[20] P. Candelas, X. C. de la Ossa, P. S. Green and L. Parkes, A pair on Calabi-Yau manifolds as an exactly solvable superconformal theory, Nucl. Phys. B 359 (1991) 21.

[21] A. Kapustin and L. Rozansky, On the relation between open and closed topological strings, Commun. Math. Phys. 252 (2004) 393 [hep-th/0405232].

[22] A. Kapustin and Y. Li, Topological correlators in Landau-Ginzburg models with boundaries, Adv. Theor. Math. Phys. 7 (2004) 727 [hep-th/0305136].

[23] D. Gepner, Exactly Solvable String Compactification On Manifolds Of SU(N) Holonomy, Phys. Lett. B199 (1987) 380. 